\title{
DUALITY MAPPING AND BIRKHOFF ORTHOGONALITY
}

BY

\section{T. PRECUPANU}

\begin{abstract}
In this note we establish some properties of Birkhoff orthogonality in terms of duality mapping. Particularly, using a slight extension of a Rockafellar result we obtain a new proof of a result earlier established by Blanco and Turnšek concerning the linear operators preserving Birkhoff orthogonality.

Mathematics Subject Classification 2010: 46B20, 46B10, 46C50.

Key words: duality mapping, Birkhoff orthogonality, subdifferential, directional derivatives, linear operator preserving orthogonality, semi-inner product.
\end{abstract}

\section{Introduction}

Let $X$ be a real linear normed space. We denote by $\perp$ the Birkhoff ortogonality generated by the norm $\|\cdot\|$ of $X$. If we consider other norm on $X$ then it generates an equivalent Birkhoff orthogonality if and only if the two norms are proportional. In [18] SсHÖPF gives a proof of this result using directional derivatives of an associated function. In 1992, KolDOBSKY [14] has proved an extension of this result to the linear operators $T: X \rightarrow$ $X$ preserving Birkhoff orthogonality. The general case of linear operators $T: X \rightarrow Y$, where $X, Y$ are two linear normed space over the field of real or complex numbers was considered by BLANCO and TURNŠEK [5]. They proved that $T$ preserves Birkhoff orthogonality if and only if $T$ is an isometry multiplied by a positive constant.

In the real case a direct proof of this result was given in [12] taking into account some properties of Birkhoff orthogonality in terms of directional derivatives of the norms. Thus, some special properties of an associated function similar to the function used by ScHÖPF [18] are obtained. 
In this paper we present some relations among Birkhoff orthogonality and duality mapping. In Section 2 we recall basic concepts and results in convex analysis concerning the subdifferential of a proper convex function. We establish a slight extension (Lemma 2) of a result of RocKAFELLAR [18], namely a sufficient subdifferential condition such that the composition of a convex function with two differential real functions to coincide up to a constant function. In the Section 3 we give some properties of Birkhoff orthogonalty in terms of duality mapping. Also, we prove that the duality mapping of a linear normed space can be defined using Birkhoff orthogonality (Theorem 5). Consequently, we obtain a new proof of Blanco-Turnšek's result (see Corollary 1 and Remarks 13 and 14).

\section{Preliminaries}

Here we recall some well-known concepts and results in convex analysis that can be found, for instance in [3], [11], [17]. Given a proper (that is there exists $\bar{x}$ such that $f(\bar{x})<\infty)$ convex function $f: X \rightarrow]-\infty, \infty]$ its subdifferential at an element $x \in X$ is defined by

$$
\partial f(x)=\left\{x^{*} \in X ; x^{*}(u-x) \leq f(u)-f(x) \text { for all } u \in X\right\} .
$$

Denote

$$
D(f)=\{x \in X ; f(x)<\infty\}
$$

called the effective domain of the function $f$. Obviously, $\partial f(x)=\emptyset$ if $x \bar{\epsilon} D(f)$.

An important property of subdifferential is the monotonicity of the multivalent operator $x \rightarrow \partial f(x), x \in X$, that is

$$
\left(x_{1}^{*}-x_{2}^{*}, x_{1}-x_{2}\right) \geq 0 \text { for all } x_{1}^{*} \in \partial f\left(x_{1}\right), x_{2}^{*} \in \partial f\left(x_{2}\right) .
$$

Moreover, this monotone operator is maximal monotone operator from $X$ to $X^{*}$. We recall that a monotone operator is said to be maximal operator if it is not properly contained in any other monotone operator.

On the other hand, for any $x \in D(f)$ there exist the directional derivatives of the convex function $f$, namely

$$
f_{ \pm}^{\prime}(x ; y)=\lim _{t \rightarrow \pm 0} \frac{f(x+t y)-f(x)}{t}, \text { for all } y \in X .
$$


If the functional $y \rightarrow f_{+}^{\prime}(x, y), y \in X$, is a linear continuous functional, then the function $f$ is said to be (Gateaux) differentiable at $x \in X$. A convex function is differentiable at any element of continuity. In fact, if $f$ is a convex function which is continuous at $x \in X$, then $f$ is differentiable at $x \in X$ if and only if $f_{+}^{\prime}(x, y)=f_{-}^{\prime}(x, y) \in \mathbb{R}$ for all $y \in X$.

Particularly, if we consider the associated real function $F_{x, y}(t)=f(x+$ $t y), t \in[0,1]$, then its right and left derivatives are just directional derivatives of $f$ :

$$
\begin{gathered}
\left(F_{x, y}\right)_{r}^{\prime}(t)=f_{+}^{\prime}(x+t y, y), \\
\left(F_{x, y}\right)_{l}^{\prime}=f_{-}^{\prime}\left(x_{0}+t y, y\right) .
\end{gathered}
$$

It is well-known that the subdifferential can be defined using the directional derivatives as following:

$$
\partial f(x)=\left\{x^{*} \in X^{*} ; x^{*}(y) \leq f_{+}^{\prime}(x ; y) \text { for all } y \in X\right\} .
$$

Also we have the following result.

Proposition 1 ([3], p.86). If the proper convex function $f$ is continuous in an element $x \in D(f)$, then

$$
f_{+}^{\prime}(x ; y)=\sup \left\{x^{*}(y) ; x^{*} \in \partial f(x)\right\} .
$$

Consequently, in this case, the set of all values of elements of $\partial f(x)$ at an element $y \in X$ is a closed segment. More precisely, we have:

$$
\left\{x^{*}(y) ; x^{*} \in \partial(x)\right\}=\left[f_{-}^{\prime}(x ; y), f_{+}^{\prime}(x, y)\right] .
$$

Now, let us consider two convex proper lower-semicontinuous functions $f_{1}, f_{2}$ such that $\partial f_{1}=\partial f_{2}$. A remarkable result of RoCKFELLAR [18] says that $f_{1}-f_{2}$ is a constant function [18]. We can obtain a slight extension of this result as follows. (For simplicity we consider the special case of continuous convex functions having nonvoid interiors of their effective domains.)

Lemma 2. Let $f_{1}, f_{2}$ be two proper convex functions which are continuous on int $D\left(f_{1}\right)$ and int $D\left(f_{2}\right)$, respectively. If $F: I \rightarrow \mathbb{R}$, is differentiable and increasing on the open real interval $I \subset \mathbb{R}$ such that

$$
F^{\prime}\left(f_{1}(x)\right) \partial f_{1}(x) \subset F^{\prime}\left(f_{2}(x)\right) \partial f_{2}(x), x \in D\left(f_{1}\right) \cap D\left(f_{2}\right) \cap f_{1}^{-1}(I),
$$

then on int $D\left(f_{1}\right)$ the functions $F \circ f_{1}, F \circ f_{2}$ coincide up to a constant function. 
Proof. Obviously, by (10) it follows that $D\left(f_{1}\right) \subset D\left(f_{2}\right)$. Let us consider two elements $x, y \in \operatorname{int} D\left(f_{1}\right)$. Therefore, we also have $t x+(1-t) y \in$ int $D\left(t_{1}\right)$ for all $t \in[0,1]$. Denote

$$
G_{i}(t)=F\left(f_{i}(t x+(1-t) y)\right), t \in[0,1], i=1,2 .
$$

By a standard calculus we obtain that these functions have right derivatives, namely

$$
\left(G_{i}\right)_{r}^{\prime}(t)=F^{\prime}\left(f_{i}(t x+(1-t) y) f_{i+}^{\prime}(t x+(1-t) y, x-y), \quad i=1,2 .\right.
$$

Now, using (9) and (10) we have

$$
\begin{aligned}
& \left(G_{1}-G_{2}\right)_{r}(t)=F^{\prime}\left(f_{1}(t x+(1-t) y) f_{1+}^{\prime}(t x+(1-t) y, x-y)\right. \\
& -F^{\prime}\left(f_{2}(t x+(1-t) y) f_{2+}^{\prime}(t x+(1-t) y, x-y)\right. \\
& =F^{\prime}\left(f_{1}(t x+(1-t) y) x_{1}^{*}(x-y)\right. \\
& -F^{\prime}\left(f_{2}(t x+(1-t) y) f_{2}^{\prime}(t x+(1-t) y, x-y)\right. \\
& =F^{\prime}\left(f_{2}(t x+(1-t) y)\left[x_{2}^{*}(x-y)-f_{2+}^{\prime}(x+(1-t) y, x-y)\right] \leq 0,\right.
\end{aligned}
$$

where $x_{1}^{*} \in \partial f_{1}(t x+(1-t) y), x_{2}^{*} \in \partial f_{2}(t x+(1-t) y)$. Consequently, the function $G_{1}-G_{2}$ is a non increasing function (see, [6], p.21). Particularly, we have $\left(G_{1}-G_{2}\right)(1) \leq\left(G_{1}-G_{2}\right)(0)$, that is $\left(F \circ f_{1}-F \circ f_{2}\right)(x) \leq$ $\left(F \circ f_{1}-F \circ f_{2}\right)(y)$, for all $x, y \in \operatorname{int} D\left(f_{1}\right)$. Therefore, we have even an equality, and so $F \circ f_{1}-F \circ f_{2}$ is a constant function on int $D\left(f_{1}\right)$, as claimed.

Remark 3. If $F(t)=t, t \in \mathbb{R}$, we obtain the result of RockAFELLAR [18] in the special case of convex and proper continuous functions.

\section{Birkhoff orthogonality and duality mapping}

We recall that the duality mapping on $X$ is the multivalued operator $J$ from $X$ to $X^{*}$ defined by

$$
J x=\left\{x^{*} \in X^{*} ; x^{*}(x)=\|x\|^{2}=\left\|x^{*}\right\|^{2}\right\}, x \in X .
$$

Remark 4. In the definition of duality mapping, the topological dual $X^{*}$ can be replaced by the algebric dual $X^{\prime}$ since according to the conditions in the right hand of (11) the continuity of any $x^{*} \in J x$ is necessarily. 
It is well-known that this multivalued operator coincides with the subdifferential of the function $f(x)=\frac{1}{2}\|x\|^{2}, x \in X$ (see, for example, [3], p.83). Therefore, the duality mapping $J$ is a maximal monotone operator on $X$. We denote the directional derivatives of this function by $n_{ \pm}^{\prime}(x, y), x, y \in X$. Also, we recall the following basic properties:

$$
\begin{aligned}
& n_{ \pm}^{\prime}(x, x)=\|x\|^{2}, \quad x \in X, \\
& n_{-}^{\prime}(x, y)=-n_{+}^{\prime}(x,-y), \quad x, y \in X, \\
& n_{+}^{\prime}(x, y) \leq\|x\|\|y\|, x, y \in X, \\
& n_{+}^{\prime}(x, \alpha x+y)=n_{+}^{\prime}(x, y)+\alpha\|x\|^{2}, x, y \in X, \alpha \in \mathbb{R} .
\end{aligned}
$$

If $n_{+}^{\prime}(x, y)=n_{-}^{\prime}(x, y), x, y \in X$, then $X$ is a smooth space. We denote by $\perp$ the Birkhoff orthogonality relation on the real linear normed space $X$, usually defined as follows

$$
x \perp y \text { if }\|x+t y\| \leq\|x\|, \text { for all } t \in \mathbb{R} .
$$

Since $t=0$ is a minimum element of the convex continuous function $t \rightarrow\|x+t y\|, t \in \mathbb{R}$, it follows that

$$
x \perp y \text { if and only if } n_{-}^{\prime}(x, y) \leq 0 \leq n_{+}^{\prime}(x, y) .
$$

On the other hand, the Birkhoff orthogonality can be characterized using the duality mapping of $X$, that is

$$
x \perp y \text { if and only if there exists } x^{*} \in J x \text { such that } x^{*}(y)=0 .
$$

Indeed, by (9) for the function $f(x)=\frac{1}{2}\|x\|^{2}, x \in X$, the statement (17) is equivalent to the statement (18).

Particularly, we have the following important property

$$
x \perp y-n_{+}^{\prime}(x, y)\|x\|^{-2} x, \text { for all } x \neq 0 .
$$

In fact, the duality mapping can be equivalently defined using the Birkhoff orthogonality.

Theorem 5. $x^{*} \in J x$ for an element $x \neq 0$ if and only if $x \perp y-$ $x^{*}(y)\|x\|^{-2} x, y \in X$ that is

$$
J x=\left\{x^{*} \in X^{*} ; x \perp y-x^{*} y\|x\|^{-2} x \text { for all } y \in X\right\}, \quad x \neq 0 .
$$


Proof. Since $\left.x^{*}\left(y-x^{*}(y)\right)\|x\|^{-2} x\right)=0$ for any $x^{*} \in J x$, then by (18) it follows that $x \perp y-x^{*}(y)\|x\|^{-2} x$.

Conversely, if $x \perp y-x^{*}(y)\|x\|^{-2} x$, using again (18) there exists $u_{y}^{*} \in J x$ such that $\left.u_{y}^{*}\left(y-x^{*}(y)\right)\|x\|^{-2} x\right)=0, y \in X$. Therefore, $u_{y}^{*}(y)=x^{*}(y)$, for all $y \in X$. Moreover, $x^{*} \in J x$. Indeed, firstly we have that $x^{*}(x)=$ $u_{x}^{*}(x)=\|x\|^{2}$. Also, $\left\|x^{*}\right\| \leq\|x\|$. On the other hand, since $x^{*}(y)=u_{y}^{*}(y) \leq$ $\left\|u_{y}^{*}\right\|\|y\|=\|x\|\|y\|$, for all $y \in X$, it follows that $\left\|x^{*}\right\| \leq\|x\|$, and so $\left\|x^{*}\right\|=\|x\|$. Consequently, by (11) we obtain that $x^{*} \in J x$. Hence, the equality (20) holds.

Remark 6. A similar result was given in [1], [8], [12].

Corollary 7. If $x, y \in X, x \neq 0$, and $\lambda \in \mathbb{R}$, then $x \perp y-\lambda x$ if and only if there exists $x^{*} \in J x$ such that $\lambda=\|x\|^{-2} x^{*}(y)$.

Remark 8. The set of all real numbers $\lambda$ such that $x \perp y-\lambda x, x \neq 0$, is a closed segment generated by the values $\|x\|^{-2} x^{*}(y), x^{*} \in J x$.

Corollary 9. If $x_{1}, x_{2} \in X$ and $\lambda_{1}, \lambda_{2} \in \mathbb{R}$ such that $x_{1} \perp\left(1-\lambda_{1}\right) x_{1}-x_{2}$, $x_{2} \perp\left(1-\lambda_{2}\right) x_{2}-x_{1}$, then

$$
\lambda_{1}\left\|x_{1}\right\|^{2}+\lambda_{2}\left\|x_{2}\right\|^{2} \geq 0
$$

Proof. By (18) there exist $x_{1}^{*} \in J x_{1}, x_{2}^{*} \in J x_{2}$ such that $\lambda_{1} x_{1}^{*}\left(x_{1}\right)=$ $x_{1}^{*}\left(x_{1}-x_{2}\right), \lambda_{2} x_{2}^{*}\left(x_{2}\right)=x_{2}^{*}\left(x_{2}-x_{1}\right)$. Since $J$ is a monotone operator on $X$, according to the definition (11) of the duality mapping we have

$$
\lambda_{1}\left\|x_{1}\right\|^{2}+\lambda_{2}\left\|x_{2}\right\|^{2}=\left(x_{1}^{*}-x_{2}^{*}\right)\left(x_{1}-x_{2}\right) \geq 0 .
$$

Now, let us consider two norms on the linear real space $X$. Denote by $\perp_{1}, J_{1}, \perp_{2}, J_{2}$ the corresponding Birkhoff orthogonalities and duality mappings, respectively. If $\perp_{1}$ and $\perp_{2}$ are equivalent it is well-known that the corresponding norms are proportional (see, for example, [5], [7], [12], [15], [19]). In earlier papers [12], [19] are given the proofs of this result using the directional derivatives. In the sequel we present an other proof in terms of duality mappings.

Theorem 10. The following properties are equivalent:

(i) $x \perp_{1} y$, whenever $x \perp_{2} y, x, y \in X$. 
(ii) The multivalued operator

$$
x \rightarrow\|x\|_{1}^{2}\|x\|_{2}^{-2} J_{2}(x), x \in X \backslash\{0\},
$$

contains the duality mapping $J_{1}$ on $X \backslash\{0\}$.

(iii) The multivalued operators

$$
x \rightarrow\|x\|_{i}^{-2} J_{i}(x), x \in X \backslash\{0\}, \quad i=1,2
$$

coincides.

(iv) There exists a positive constant $k>0$ such that $\|x\|_{1}=k\|x\|_{2}$, for all $x \in X$.

Proof. (i) $\Rightarrow$ (ii) Let us consider $x^{*} \in\|x\|_{1}^{-2}\|x\|_{2}^{2} J_{1}(x), x \neq 0$. Therefore, we have $\|x\|_{1}^{2}\|x\|_{2}^{-2} x^{*} \in J_{1}(x)$, and so, by Theorem 4 for every $y \in X$ we get

$$
x \perp_{1} y-\|x\|_{2}^{-2} x^{*}(y) .
$$

Consequently, by (i) we also have

$$
x \perp_{2} y-\|x\|_{2}^{-2} x^{*}(y) .
$$

Now, using again Theorem 4 we obtain that $x^{*} \in J_{2}(x)$.

(ii) $\Rightarrow$ (iii) Using the properties of duality mapping we can prove that the multivalued operator $\widetilde{J}$ defined by $\widetilde{J}(x)=\|x\|_{2}^{-2}\|x\|_{1}^{2} J_{2}(x), x \in X \backslash\{0\}$ is a monotone operator. Taking by definition $\widetilde{J}(0)=0$, this operator monotone contains the maximal monotone operator $J_{1}$. Therefore, $\widetilde{J}=J_{1}$ and so, $J_{1}(x)=\|x\|_{2}^{-2}\|x\|_{1}^{2} J_{2}(x)$, for all $x \in X \backslash\{0\}$.

(iii) $\Rightarrow$ (iv). By Lemma 2 for $f_{1}=\frac{1}{2}\|\cdot\|_{1}^{2}, f_{2}=\frac{1}{2}\|\cdot\|_{2}^{2}, F(t)=\ln t, t>0$, we obtain that $\ln \|x\|_{1}=\ln \|x\|_{2}+C, x \neq 0$, where $C$ is a constant function. Consequently, $\|x\|_{1}=k\|x\|_{2}, x \in X$, for a certain constant $k>0$.

Corollary $11([5])$. A linear operator $T: X \rightarrow Y$, where $X, Y$ are two real linear normed spaces endowed with the norms $\|\cdot\|_{X},\|\cdot\|_{Y}$, preserves Birkhoff orthogonality if and only if it is an isometry multiplied with a positive constant.

Proof. Firstly, we remark that $\|x\|_{T}=\|T x\|, x \in X$, is a norm on $X$, whenever $T$ is a linear operator preserving Birkhoff orthogonality (see 
[5], [12]). But, if $T$ preserves Birkhoff orthogonality, then the Birkhoff orthogonality on $X$ implies the Birkhoff orthogonality generated by the norm $\|\cdot\|_{T}$. Thus, we apply the Theorem 10 for the norms $\|\cdot\|_{X},\|\cdot\|_{T}$ on $X$.

Remark 12. The duality mapping with respect to the norm $\|\cdot\|_{T}$ may be defined as follows:

$$
J_{T} x=\left\{x^{*} \in X^{\prime} ; x^{*}(x)=\|T x\|^{2}, x^{*}(y) \leq\|T x\|\|T y\|, \text { for all } y \in X\right\} .
$$

Consequently, we can reformulate the statements (ii), (iii) in Theorem 10 replacing $J_{1}$ by $J_{X}$ and $J_{2}$ by $J_{T}$, respectively.

Remark 13. Now, let $X$ be a complex linear normed space. If we denote by $\perp_{C}, \perp_{R}$ the Birkhoff orthogonality with respect to $\mathbb{R}$ and $\mathbb{C}$, respectively, then $x \perp_{C} y$ is completely defined by $x \perp_{R} y$ and $x \perp_{R} i y$. Consequently, the equivalence (i) $\Leftrightarrow$ (iv) in Theorem 10 also holds in any complex linear normed space $X$.

Remark 14. Let $[\cdot, \cdot]: X \times X \rightarrow \mathbb{R}$ be a semi-inner product inducing the norm on $X$, i.e., $\|x\|=[x, x]^{1 / 2}$, for any $x \in X$. We recall that a semiinner product have all properties of a inner product except the symmetry property which is replaced by Cauchy-Buniakowsky inequality (for detailed definition of semi-inner product see [10], [16], [17]). It is well-known that a semi-inner product is generated by the selections of duality mapping. Indeed, if $s$ is a selection of duality mapping $J$, then $[x, y]=(s(x))(y)$, $x, y \in X$, is a semi-inner product and conversely. Thus, if in Theorem 10 the statement (i) is replaced by

$\left(\mathrm{i}^{\prime}\right)[x, y]_{1}=0$, then $[x, y]_{2}=0$,

then (ii) becomes

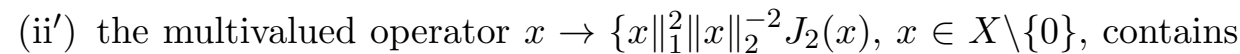
the selection $s_{1}(x), x \in X \backslash\{0\}$, generated by semi-inner product $[\cdot, \cdot]_{1}$ on $X$.

But, since $J_{1}$ and $J_{2}$ are maximal monotone operators the statements (ii) and (ii') are equivalent. Consequently, (i') and (iv) are also equivalent in the case of orthogonality generated by two inner-product on $X$. 
Generally, if two elements are orthogonal with respect to any semi-innerproduct on $X$ which generates the norm of $X$, then these elements are also Birkhoff orthogonal. Conversely, if two elements $x, y$ are Birkhoff orthogonal there exists a semi-inner-product $[\cdot, \cdot]$ such that $[x, y]=0$. If the space is smooth [3], [9], [11], [17], then there exists an unique semi-inner-product, and so, $x \perp y$ if and only if $[x, y]=0$.

\section{REFERENCES}

1. Alonso, J.; Martini, H.; Wu, S. - On Birkhoff orthogonality and isosceles orthogonality in normed linear spaces, Aequationes Math., 83 (2012), 153-189.

2. AmiR, D. - Characterizations of Inner Product Spaces, Operator Theory: Advances and Applications, 20, Birkhäuser Verlag, Basel, 1986.

3. Barbu, V.; Precupanu, T. - Convexity and Optimization in Banach Spaces, Fourth Edition, Springer, 2012.

4. Birkhoff, G. - Orthogonality in linear metric spaces, Duke Math. J., 1 (1935), 169-172.

5. Blanco, A.; TuRnšEK, A. - On maps that preserve orthogonality in normed spaces, Proc. Roy. Soc. Edinburgh Sect. A, 136 (2006), 709-716.

6. Bourbaki, N. - Fonctions d'une variable réele, Chap. 1, 2 et 3, Hermann, Paris, 1958.

7. Chmieliński, J. - Linear mappings approximately preserving orthogonality, J. Math. Anal. Appl., 304 (2005), 158-169.

8. ChMieliński, J. - Remarks on orthogonality preserving mappings in normed spaces and some stability problems, Banach J. Math. Anal., 1 (2007), 117-124.

9. DAy, M.M. - Normed Linear Spaces, Springer-Verlag, Berlin-Göttingen-Heidelberg, 1958.

10. Giles, J.R. - Classes of semi-inner-product spaces, Trans. Amer. Math. Soc., 129 (1967), 436-446.

11. Holmes, R.B. - Geometric Functional Analysis and its Applications, Graduate Texts in Mathematics, No. 24, Springer-Verlag, New York-Heidelberg, 1975.

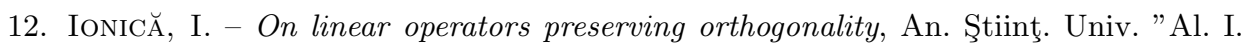
Cuza" Iaşi. Mat. (N.S.), 58 (2012), 325-332.

13. James, R.C. - Orthogonality in normed linear spaces, Duke Math. J., 12 (1945), 291-302. 
14. James, R.C. - Orthogonality and linear functionals in normed linear spaces, Trans. Amer. Math. Soc., 61 (1947), 265-292.

15. Koldobsky, A. - Operators preserving orthogonality are isometries, Proc. Roy. Soc. Edinburgh Sect. A, 123 (1993), 835-837.

16. Lumer, G. - Semi-inner-product spaces, Trans. Amer. Math. Soc., 100 (1961), 29-43.

17. Precupanu, T. - Linear Topological Spaces and Fundamentals of Convex Analysis (Romanian), Editura Academiei Române, Bucharest, 1992.

18. RockAfEllar, R.T. - On the maximal monotonicity of subdifferential mappings, Pacific J. Math., 33 (1970), 209-216.

19. SсHÖPF, P. - Orthogonality and proportional norms, Anz. Österreich. Akad. Wiss. Math.-Natur. Kl., 133 (1996), 11-16 (1997).

Received: 30.V.2011

Department of Mathematics,

University "Al.I. Cuza", 11, Bd. Carol I, 700506, Iaşi,

ROMANIA

tprecup@uaic.ro 